\title{
Upper Limb Rehabilitation System for Self-Supervised Therapy: Computer-Aided Daily Performance Evaluation for the Trauma and Disorder in the Spinal Cord and Peripheral Nerves
}

\author{
Kengo Ohnishi, Keiji Imado**, Yukio Saito* \& Hiroomi Miyagawa** \\ Okayama Prefectural University, *Tokyo Denki University, ${ }^{* *}$ Oita University
}

Japan

\section{Introduction}

With the serious impact on life caused by the disability after cerebrovascular accidents and other serious trauma of the nervous system, prevention strategies and survivors' rehabilitation programs are one of the social needs in many countries. As in super-graying society with low death and birth rate like Japan, an approach of just fostering aid personnel with necessary expertise will not be satisfactory to ensure the quality of the care services. Research in therapeutic technique needs to be paralleled by technology development to reduce the load on the care givers and medical staffs, as well as the development of devices that directly assists the survivors' independent living. Furthermore, advanced efforts are made in Japan to generally decentralize healthcare from clinics to homes and local communities. The escalating healthcare service need to be halted, and strategies such as $\varepsilon$ shortening the patient's hospital stay by reducing the time spent in bed and maximizing the o time spent for rehabilitation with the support of technology. To meet our goals, further $\Phi$ scientific research is required to understand the factors for enhancing the rehabilitation effects. This will reduce the hands-on one-on-one treatment so that the therapy programs 드 can be provided in homes and local clinics without full-time supervision. With the 응 promising achievements in rehabilitation robotics, we believe further studies on sensor-

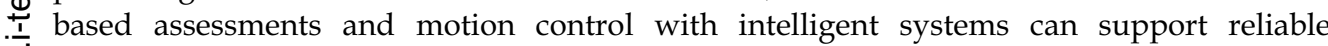
advancements of the therapy programs.

In this chapter, our work on developing a rehabilitator system for cervical spinal cord injured

$£$ clients is presented. Our main aim is to provide post-hospital-discharge clients with opportunity

$\mathbb{N}$ for voluntary training and to assist self-supervised therapy. Automatic recording, adaptive 을 exercise control, and multilateral analysis are the key technology that we target for recruiting and $\widetilde{T}$ assisting the client's rehabilitation and medical staff's interest in using the device.

\section{Target Population and Rehabilitators}

\subsection{Cervical Spinal Cord Injury and Therapy}

The incidence of Spinal Cord Injury (SCI) lies between 10.4 and 83 per million inhabitants per year by (Wyndaele et al., 2006). One-third of the patients with SCI are tetraplegic and 
$50 \%$ of patients with SCI have a complete lesion. The mean age of patients sustaining their injury is 33 years-of-age, and the sex distribution is 3.8 male per female. Shingu and Sumida (Shingu et al., 1995; Sumida et al., 2001) report the characteristics of Japan. The incidence is 40.2 per million inhabitants per year, which yeilds 5,000 new people per year and 80,000 patients are alive. The statistics on the age of injury for SCI in Japan is different from those with single-peak distribution of the European countries and USA. The Japanese figure is a bimodal distribution with peaks at the age of 20 and 59. From the same statistics, the number of Cervical Spinal Cord Injuries (CSCI) out numbers the thoracic and lumber spine injuries after the age of 40 . Furthermore, the rate of CSCI goes up to $88 \%$ for those aged over 65. From these figures, it is clear that rehabilitation programs are needed for the new patients at greater age. Another problem is seen in the senior CSCI clients that 15 to 20 years had passed after their injury (Takaoka et al., 1999). Their functional ability declines due to muscular weakness, being easily-fatigued, and pain which are age-related. These clients need nearly full nursing care. And with lower activity, secondary medical condition can be developed to complicate their condition more and restrict the participation to rehabilitation program.

From these facts, rehabilitation for the senior clients is as critical as those for the young injured clients in Japan. The major problems for the senior population are insufficient amount of medical rehabilitation for the acute clients, and strong depression that weakens the motivation to the participation to rehabilitation program for the chronic clients (Takaoka et al., 1999). We believe that both group would benefit from using an easy-to-use therapy device to retrain or maintain their physical ability. As (Lenze et al., 2004) reports, if senior patient's good participation in physical and occupational therapy shortens the length of stay in the hospital, it would be favourable for the clients to use a device during their stay and then take home the same system. That way, the program will be consistent and the clients would have a better understanding of how to use the device and know how to assess their performance after the discharge. Furthermore, if a regular exercise regime would help to control pain as (Latimer et al., 2004) reports, recordings of the exercise session may help the medical professional to gain further understanding of the pain symptom and other comorbidity of SCI.

\subsection{Rehabilitators}

Many upper limb rehabilitation devices applying robotics and mechatronics technology are developed. Just to name a few devices, they are Massachusetts Institute of Technology Manus (Krebs et al., 1998), Mirror Image Motion Enabler (Shor et al., 2001), Assisted Rehabilitation and Measurement Guide (Kahn et al.; 2001), GENTLE/s (Amirabdollahian et al., 2001; Coote et al., 2003), and Exercise Machine for Upper Limb (Furusho et al., 2003), These rehabilitators are designed to mainly treat stroke patients' hemiparetic proximal upper limb by passively or partially assisting the motion of the subject (Reinkensmeyer, 2003; Reinkensmeyer et al., 2000). Prange reports on a systematic survey on the clinical results of these rehabilitators (Prange et al., 2006). Due to the limited number of participants in the reviewed papers and their follow up studies, it is still difficult to draw a firm conclusion of the impact of the rehabilitators. However the results show better positive improvements compared to conventional methods in many cases.

These systems with robot assisted rehabilitation enables to provide services with accuracy and repeatability, while recording the performance with quantitative measure. However, robotic system faces difficulty of implementing safety feature requirements to properly use 
the robot's movability. To reduce the risk at operation, skilled operator needs to be at site to observe the proper process flow and to react on emergency. In addition, further research is necessary to discuss the control method and appropriate set up for the robotic arm to assist the motions and forces to satisfy positive effect of use which a trained therapist will normally do in their program. For robotic rehabilitation, Hogan states based on their research results that 1 ) muscle strengthening offers no advantage over movement training, 2) passive movements is insufficient and active participation is required, and 3) progressive training based on measures of movement coordination yields substantially improved outcomes (Hogan et al., 2006). Moreover, space, cost, and usability are the decisive factors of purchasing such systems to be used in local clinics or homes. Financial aid as well as technical aids will be needed to have such device widely used.

In addition, the applicant of rehabilitation robot is currently focused on stroke patients. SCI is also known to cause a major physical disability from nerve damage. Peripheral nerve damage in upper limb also strongly affects the hand function and paralyses the digit movements. The lesion of the stroke patient is in the cortical area where the plasticity of the brain will play an important role in the recovery of the functional ability, whereas the lesion of the spinal cord and peripheral nerves are damage to the signal transmission line network. Recovery such as nerve regeneration and reinnervation process may need surgical procedure and will take longer time to regain the function. Therefore, the outcome and methodologies of the stroke rehabilitation may not be fully applied to the rehabilitation strategies for the SCI. Studies on Functional Electrical Stimulation (Popovic et al., 2002; Haugland et al., 1999) for the SCI is also a field where researches are ongoing to obtain joint movements in the upper limb. Their results and other exercise therapy assessment methods should be mutually beneficial. Assessment is one area that all fields can have good information exchange and new assessment technique will contribute in understanding the observable fact in multifaceted approach.

\section{Method}

\subsection{Design Concept and System Structure}

Our objective is to develop a device that CSCI clients and medical professionals can use to do the routine exercise at home or neighbouring local clinic, and to evaluate the momentary and temporal changes of the performance. The first goal was to create a prototype hardware and a test-bed training program to run a small field test. Our objective for this study was to confirm whether the device can be used in local clinics without engineer or technician at site, and whether the therapists can recruit outpatients to use the device by themselves on regular bases. With few steps of modification, the device has assisted to encourage a subacute CSCI patient and a chronic CSCI patient with severe depression to use the device to prepare themselves to restart the therapy which they were first a poor participant. Also, a young peripheral nerve injured patient was keen to use the device almost every day over 6 months period. With these small but encouraging successful experiences and constructive data, our next and current goal is to propose an evaluation method that is easy to understand and capable of encouraging the subjects to maintain their exercising routine. Our device had encouraged the subject to work out when the subjects where in a stage where they were still very physically and mentally weak and unprepared for regular therapy programs. However, as the subjects became more active and less physically limited, the exercise and performance scores were unchallenging, monotonous, and unattractive. A 
more sensitive measure such as feature extraction method that can analyse the data multilaterally with minimum processing load is in need. The ambition of the future system is to automatically or interactively feedback the performance data to the program parameters as in video games and enhance the training effect. This will be our future goal which are discussed later.

\subsection{Hardware, Software and Operation}

The system consists of a liquid crystal display, a personal computer, interface peripherals, and a joystick-type controller (See Fig. 1). The first prototype controller consists of a grip, joystick module, buttons. The controller has a plastic stage that can be fitted on the laptop of a subject sitting on an adult-size wheelchair or on the device's computer desk. The second prototype is redesigned to be used on desktop and the buttons are omitted by modifying the software to run and respond to conditions automatically. The joystick is a stiff rod and does not have tilt angle as in video game joystick controllers. A tri-axial load cell is developed and installed in the joystick to measure the exerted force. The strain gauge is mounted on the load cell and the amplifier circuit is installed in a case under the stage. Amplified signals from the strain gauges are scanned by 12-bit A-D converter board (Interface Co., Model: PCI- 3171A, Japan). The sampling frequency is $5 \mathrm{kHz}$. This is conditioned to reduce the rag of marker movements on screen while smoothing the signal with moving-average method. Each of the sampled signals in the horizontal plane is integrated and compared with the initial condition values. Then the differences are assigned to a quadratic function to compute the marker travel distance, $d X, d Y$. The marker travel distance for $d Z$, which is shown as a floating bar on the screen, is processed in proportion to the difference value.

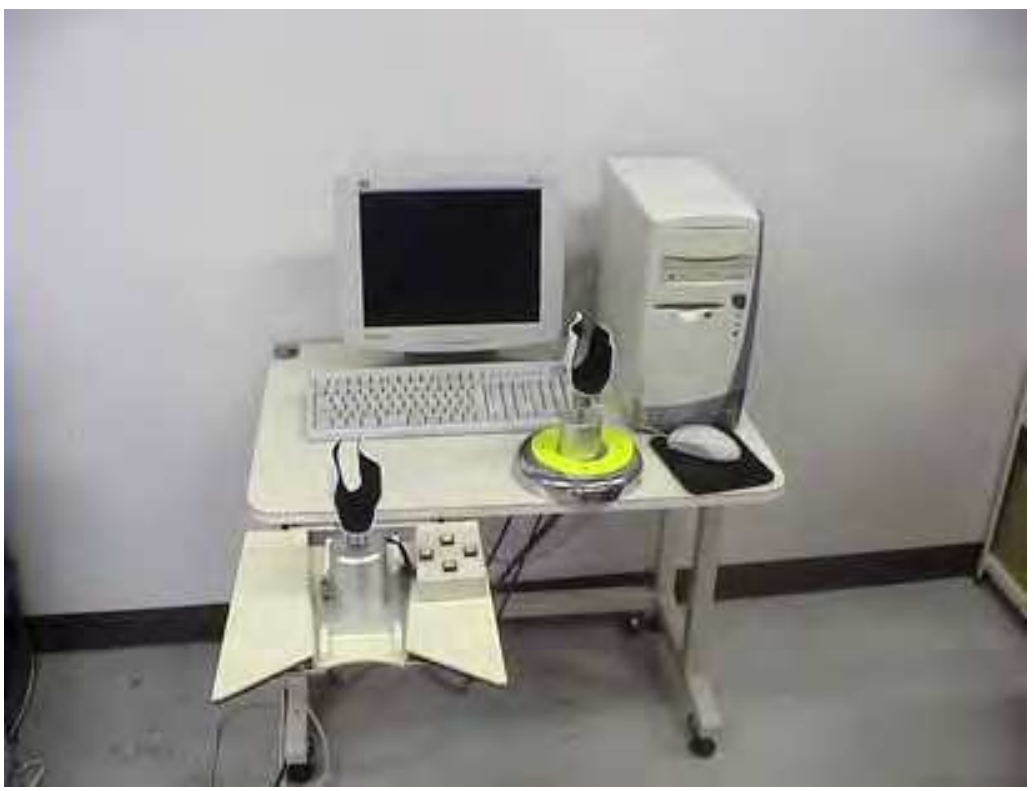

Fig. 1. The first and second prototype of upper limb rehabilitation system for spinal cord and peripheral nerve injury. The first prototype in the front left is mounted on a stage to place on the laptop. The second prototype is for desktop use. 
The system operation is like a video game. A circle and a flower shape trajectory are arranged for exercise and evaluation menu (See Fig. 2). Sagittal plane is set as the Y-axis (vertical axis) on the trajectory, and the frontal plane as the $\mathrm{X}$-axis (horizontal axis). A red circle target and a green square marker are presented on the screen. The target on the trajectory moves at a constant angular speed, counter-clockwise from the initial position on the positive X-axis. The subject's goal is to maintain the marker within minimum distance to the target center. The subject controls the marker by applying force to the joystick based on the visual information of the trajectory and target-marker distance. The system diagram of the operation is shown in Fig.3. The exercise session is followed by a one-minute presentation of the performance result. The locus of the marker and score are computed from the recorded performance data and presented on the screen.
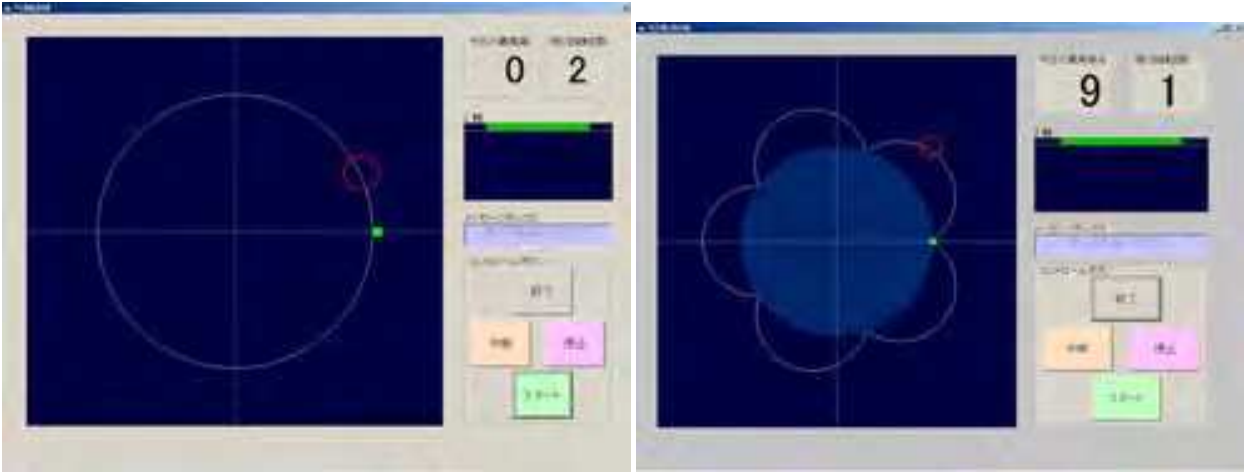

Fig. 2. Two trajectory, circle and flower shape, are created for the exercise screens. The red small circle on the trajectory is the target which is followed by the green square marker that the subject operates. Forces applied to the joystick in sagittal and frontal plane propels the marker in $\mathrm{X}-\mathrm{Y}$ coordinate. The bar in the middle right represent the force applied to the vertical axis and moves like a floating stick with buoyancy.

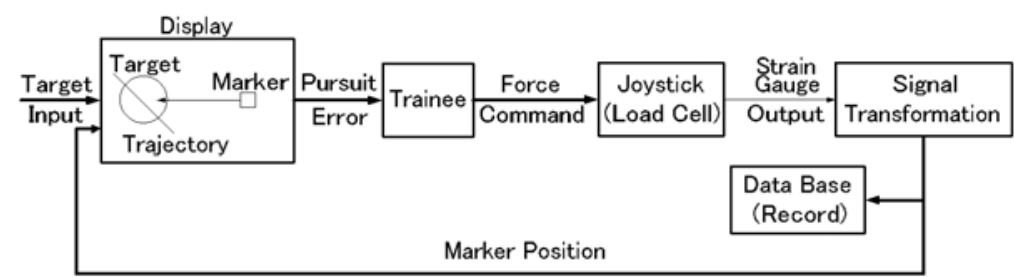

Fig. 3. The system diagram of the operation scenario.

\subsection{Performance Indices}

Many performance indices are proposed to evaluate the recorded upper limb operation data. Krebs' group reported their two categories of performance indices based on the type of humanmachine interaction, with and without robot assistance (Krebs et al., 2001). Hand path displacement, lateral deviation of subject's movement from a straight line connecting the targets, and other 4 indices are calculated as the unconstraint indices. Mean force, speed, and power are calculated during the robot assistance as the constraint indices. Furusho's group uses positioning deviation in time series to discuss their subject's performance changes as the training proceeds (Furusho et al., 2003). Takahashi's group described the subject group's characteristics by 
describing the data in relation of the time required for the task to the sum of square of error distance (Takahashi et al., 2003). Chelette's group compared the sensitivity of time domain parameters and phase plane parameters as metrics of performance. The time domain parameters showed usefulness in distinguishing subject ability groups, however they recommend error phase plane parameters because of their higher sensitivity to the effect of special force reflection (Chelette et al., 1995). Ide's group proposed four phases in cursor operation; response time, approaching time, adjusting time, and switching time (Ide et al., 1995). With total time, number of inching operation, and the four indices, they described the characteristic of the CSCI patients' joystick operation. Murayama developed a system that uses a stylus pen and a monitor with a built-in digitizer to evaluate the performance of drawing circular trajectory (Murayama, 2002). For analysis, he proposed mean velocity, acceleration, and power spectrum of the squared residual error to characterise the difference of the subjects and the effect of rehabilitation.

As in these earlier studies, we also used residual error distance in time domain as our main evaluation index. The marker locus $M$ is calculated by summing $d X$ and $d Y$ in time sequence, as in Equation (1). The error distance is calculated as the distance of the marker position $\boldsymbol{M}$ and the target position $T$ as in Equation (2).

$$
\begin{aligned}
X_{M}(t)= & \sum_{n=0}^{t} d X(n)+X_{0}, \quad Y_{M}(t)=\sum_{n=0}^{t} d Y(n)+Y_{0} \\
E(t)= & \sqrt{\left\{X_{T}(t)-X_{M}(t)\right\}^{2}+\left\{Y_{T}(t)-Y_{M}(t)\right\}^{2}} \\
& \left\{M \mid X_{M}(t), Y_{M}(t)\right\}: \text { Marker position, } \\
& \left\{T \mid X_{T}(t), Y_{T}(t)\right\}: \text { Target position, } \\
& E(t): \text { Error distance } \\
& t: \text { sampled time }
\end{aligned}
$$

Since our target tracking scenario exercises a steady amplitude force generation in all circumference direction, the distribution of the control signal is also assessed. By plotting the forces in $\mathrm{F}_{\mathrm{x}}-\mathrm{F}_{\mathrm{y}}$ coordinate and discussing the density of the plotted points, the result in the early stage of the experiment showed wider distributed pattern, whereas the latter were congregated to a relation of sum of $\left|F_{x}\right|$ and $\left|F_{y}\right|$ being constant.

As mentioned previously, the momentary target-marker distance is a common measure for discussing the performance. This error in time series describes the subject's continuous positioning error which is an accumulated response delay. Therefore, the result is strongly influenced by the response at the starting point and the ratio between target speed and the gain of marker displacement. The result is influenced by the feedfoward characteristic during the learning curve which will diminish when the target speed is constant. The performance characteristic can be further studied by presuming a control system and identifying the parameters in a model-based approach. Furthermore, the performance can be studied in space, frequency or non-dimensional domain instead of discussing it in time or time-space domain. In this study we chose to model the geometrical characteristic to discuss the precision. Since our target trajectory is based on circles, we implemented a method used in measuring the roundness to discuss the position error as next.

When considering the target trajectory as a perfect circle, the marker locus can be presumed as distorted circle. That is, the marker locus can be identified as a sinusoidal waveform with distortion component that can be expressed as periodic function. Consequently, harmonic analysis (Nakata, 1972) is implemented to model the performance locus. On identification, the 
center of target trajectory coordinates $\mathrm{O}$ is set as the center of roundness measurement. When the marker locus is described as $\mathrm{K}$ and the center of the target trajectory as $\mathrm{O}$, a norm circle $\mathrm{M}$ is computed. $\mathrm{M}$ is a circle with an center $\mathrm{O}^{\prime}$ and radius $a_{0}$. See Fig. 4 for their relation. By describing the displacement of center $\mathrm{O}^{\prime}$ in polar coordinates as $\left(c_{1}, \phi_{1}\right)$, locus $\mathrm{K}$ is expressed as Equation (3) through (7). The coefficient of the right end term in Equation (3) is insignificant when $n$ is significant, and consequently, $n$ can be agreed as a finite number. In our algorithm $n$ $=24$ is applied, therefore 24 equally divided harmonic analysis is used to calculate the norm circle's eccentric relation $\left(c_{1}, \phi_{1}\right)$ and $a_{0}$. Here, the compared distortion $\varepsilon(\theta)$ is the subtraction of the norm circle $\mathrm{M}$ from the locus $\mathrm{K}$, as Equation (8). We equally divided the circle in 24 segments so the compared distortion is computed as $\varepsilon_{p}$ at the selected 24 point on the circle.

$$
\begin{gathered}
K: k(\theta)=a_{0}-E+a_{1} \cos \theta+b_{1} \sin \theta+\sum_{n=2}^{\infty}\left(a_{n} \cos n \theta+b_{n} \sin n \theta\right) \\
a_{0}-E=\frac{k_{1}+k_{2}+\cdots+k_{23}+k_{24}}{24} \\
a_{1}=\frac{k_{1} \cos (\pi / 24)+k_{2} \cos (\pi / 12)+\cdots+k_{23} \cos (23 \pi / 24)+k_{24}}{12} \\
b_{1}=\frac{k_{1} \sin (\pi / 24)+k_{2} \sin (\pi / 12)+\cdots+k_{23} \sin (23 \pi / 24)}{12} \\
\varepsilon(\theta)=\varepsilon_{p}=k_{p}-\left\{a_{0}+a_{1} \cos (p \pi / 24)+b_{1} \sin (p \pi / 24)\right\} \\
\because p=1,2, \cdots, 24
\end{gathered}
$$

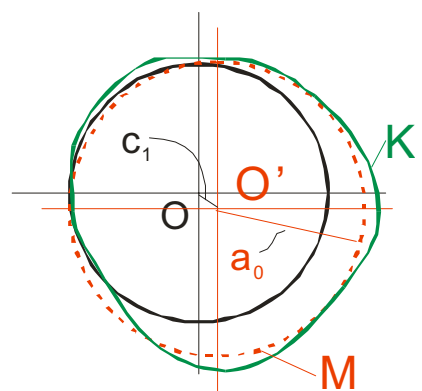

Fig. 4. Diagram of the norm circle and parameters in the harmonic analysis.

The recorded data is calculated offline into the four variables $c_{1}, \phi_{1}, a_{0}$, and $\varepsilon_{p}$. First, the marker locus $L:[x, y]$ is computed by summing the marker displacements for unit time, $\Delta x_{t}$ and $\Delta y_{t}$, and then transformed to polar coordinate $L_{p}:[k, \theta]$. The radius of the target trajectory $\mathrm{E}$ is normalized $(=1)$, and the marker locus $\mathrm{K}$ is transformed accordingly. On selecting the $k_{P}$ from the converted marker locus data, the value corresponding for $\theta$ with the closest acceptable approximation to $n_{p}(=0,15,30, \ldots, 360$ degrees $)$ is chosen. We used 0.5 degrees in the algorithm for processing the locus that is virtually a circle. When no candidate is found, acceptable range is expanded. The algorithm will halt with an error in case where the marker locus does not close as a circle and forms a C-shape. This occurs when the subject drops greatly behind the target and the final position of the marker does not come near to the starting point before the unit 
session time is up. In such case, the value for the $\theta$ closest to 360 degree is chosen to fill in the data. Furthermore, the marker locus over wraps when the marker over runs the target and reverses. In such locus, $k_{p}$ is chosen from the most neighboring value to $n_{p}$.

\section{Field Test and Evaluation Results}

All subjects were tested following informed consent. Twelve able-bodied subjects $\square 8$-male and 4-female, average age 22.6, Std. Dev. 0.99 $\square$ participated in the pilot test for creating the database and to confirm the validity of the index and algorithm. The two CSCI subjects' data was then applied to computation. These data was collected after approval of the study from the Institutional Review Board of each hospital that the subjects were receiving their medical treatment. Informed consent was given from the therapist before the test. The first subject was a senior male ambulatory patient and the trials were made at his presence to the clinic on his pace. This subject was paresis and capable of walking but had numbness in both arms at the beginning of the test. The numbness had decreased to a level that there was no discomfort when the subject withdrew from the test. The second subject was a senior male hospital patient. He was a quadriplegic and both arms were very weak and the voluntary ranges of motion of the upper limb joints were limited to minimal. He had strong depression and needed personal care for all activity at the beginning of the test, but regained partial strength in the shoulder by the end of first 4 weeks of the test. Both patients had returned to the clinic reporting decrease in their upper limb sensory-motor function and to reapply for a treatment. The target speed was set to $1.0 \mathrm{rpm}$. The controller was set on the side table and the hand position was adjusted to suit each patient. The first CSCI subject had four sets of each circle and flowerpattern trajectory pattern as routine exercise session and the second CSCI subject had two of each pattern. Testing began after the patient understood how to control the marker on the screen by practicing on the demonstration mode and the gains in the controller were adjusted for each subject at the beginning of the test. No modifications were made to the condition through the whole trials. Ten days of data was recorded between a 49-days period for the first subject, and 18 days of data was recorded between an 85-days period. The first and tenth day's data of both subjects were evaluated with the proposed indices. The marker loci of the two subject's last trial in their session are shown in Fig. 6. The target-marker error distance are described in Fig. 7.

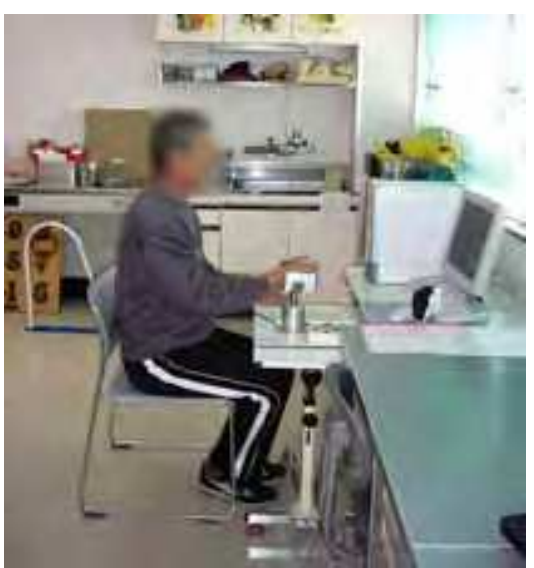

Fig. 5. The first CSCI subject working on his routine program on the device. 


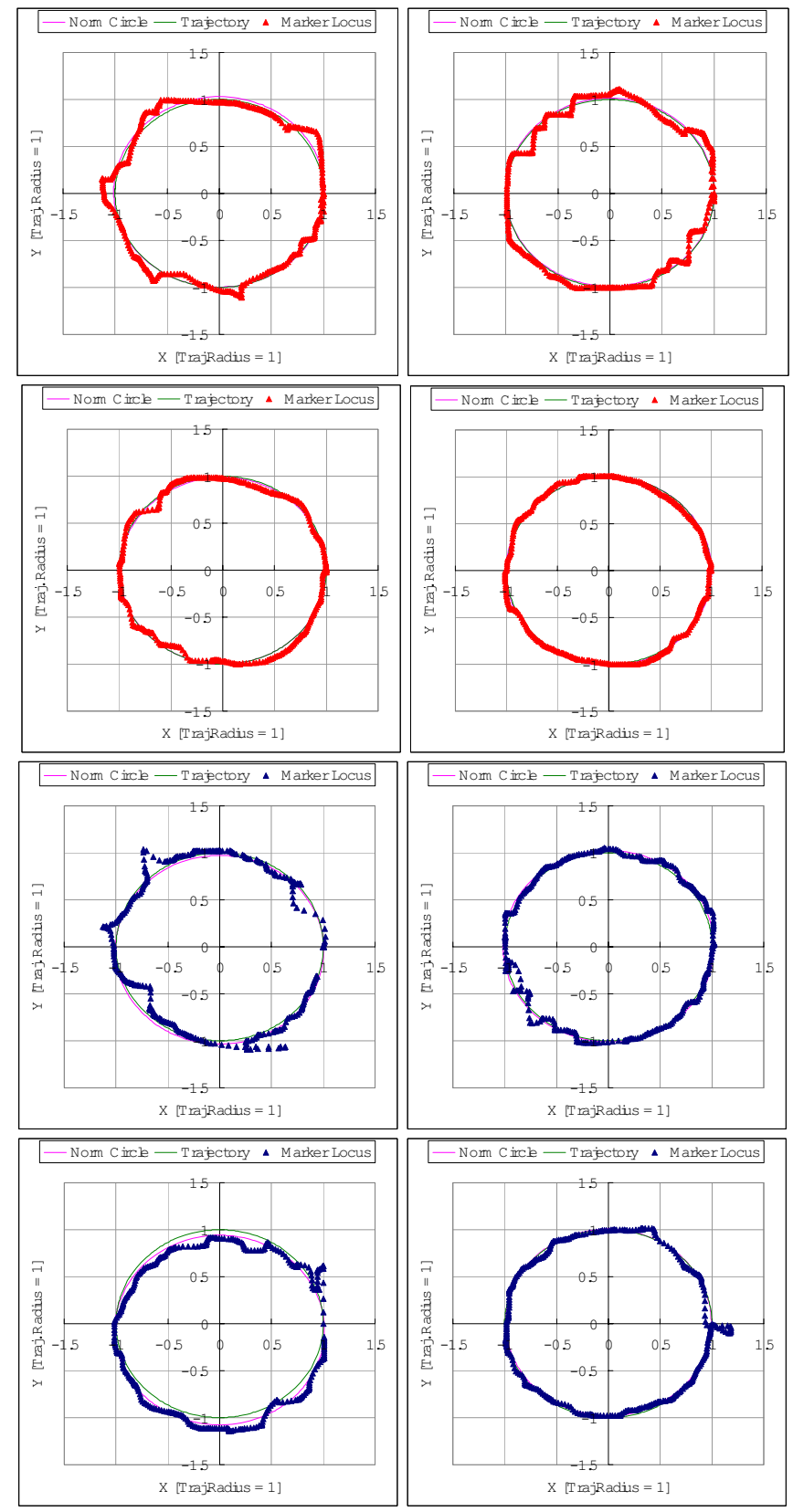

Fig. 6. The diagram of the $1^{\text {st }}$ (top 4) and 2nd (bottom 4) CSCI subject's marker loci and norm circles of each trial. The $1^{\text {st }}$ and $3^{\text {rd }}$ row are their $1^{\text {st }}$ day's, and $2^{\text {nd }}$ and $4^{\text {th }}$ row are their $10^{\text {th }}$ day's data. The graphs in the left column are the first and the right column are their last trials of each day. 


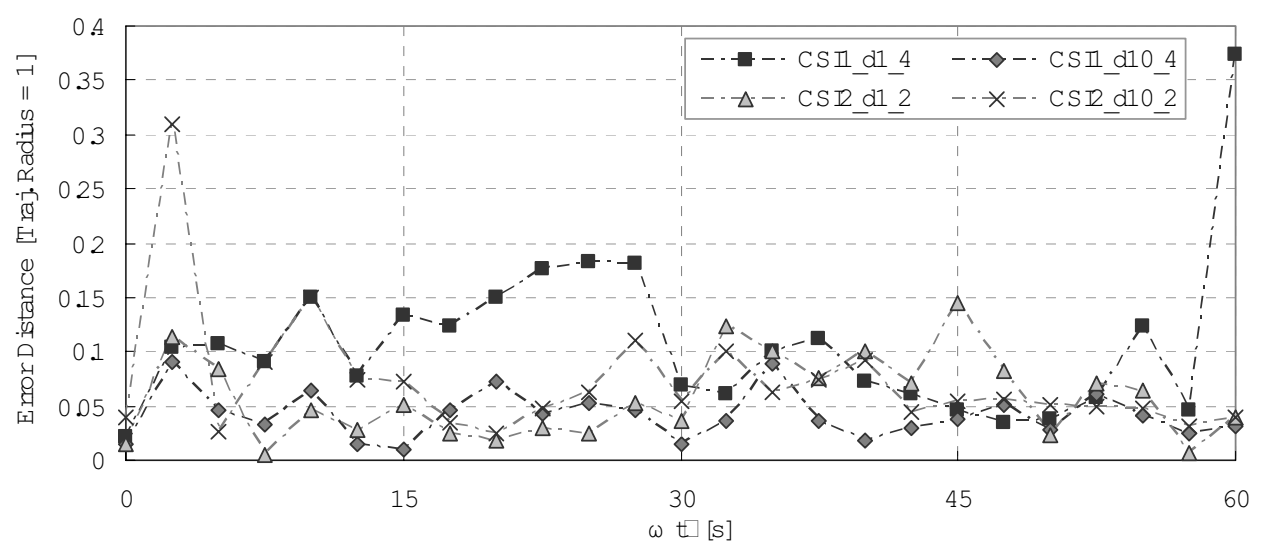

Fig. 7. The variance of error distances in time series. The first and second CSCI subject's data are presented as CSI1 and CSI2, accordingly. The $1^{\text {st }}$ day's data is shown as d1 and 10 $0^{\text {th }}$ day's data as d10. The data are all last trials of the day.

\section{Discussion}

The first CSCI subject's performance on the first day is shown at the top row of Fig. 6 . As seen in the right diagram's locus, the track is relatively square. This illustrates that the direction of the marker was only changed in a while. The error distance for this session's result drawn in Fig. 7 are higher then the other 3 results. When interpreting these information from the graphs, you can picture that the subject was having difficulty following the target. However, it is difficult to comment the major cause of the error was whether he was running behind the target or off the trajectory. Also the upsurge at the end of the trial in Fig. 7 cannot be found on the locus of top right diagram of Fig. 6. For the second subject the dislocation at the start on the tenth day's locus in the bottom right diagram of Fig. 6 can be confirmed as the peak at the left end in Fig. 7. The immediate decrease of error distance in Fig. 7 shows that subject had instantly caught up to the target. However, there is another rise of error around $30 \mathrm{~s}$ in Fig. 7. For the first day, the cause is running off the trajectory seen as the wavering in the diagram of Fig. 6. However, since no major wavering of the locus is seen in the bottom right diagram of Fig. 6, it is assumed that the cause of the 10th day's result is delay.

To confirm the error in space domain, the variables $c_{1}, \phi_{1}, a_{0}$, and $\varepsilon_{p}$ are computed. The results of $a_{0}$ are described in Fig. 8, the eccentric relation in Fig. 9, and $\varepsilon_{p}$ in Fig. 10. It is shown in Fig.8. that all error of the norm circle radius is less than $2 \%$ of the target trajectory's radius. Furthermore, the variations of the radius within a day are smaller on the tenth day for both subjects. From Fig. 9, it shows that the majority of the dislocated center remain within the $2 \%$ range of the $c_{x}-c_{y}$ coordinates. Compared with this, the second subject's first session's result are located exceedingly to the negative side in the $c_{y}$ direction. Furthermore, both CSCI subject's results show that the distance from the center had decreased in their tenth day's session. In Fig. 10, the maximum distortion had decreased in the 10th day's data for both subjects. These diagrams interpret the strategies of following the 
target in a quantitative manner. The points inside the 0 describes that the subject is taking a shorter path and moving the marker with reduced effort to track the target. If the error distance is small and the distortion is negative as in the top-left area $(t=15-30 \mathrm{~s}, n=6-12)$ of the second-subject's first-day data, the subject is presumed to be relaxed and composed of following the target. In contrast, when the error distance is large and distortion is negative as in the bottom-left area $(t=30-45 \mathrm{~s}, n=12-18)$ of the second-subject's tenth-day's data, the subject is presumed to have difficulty following the target and learned to take a shorter path to compensate the delay. Consequently, the geometrical information is quantitatively comparable and feature are extracted with these indices for further understanding of the performance.

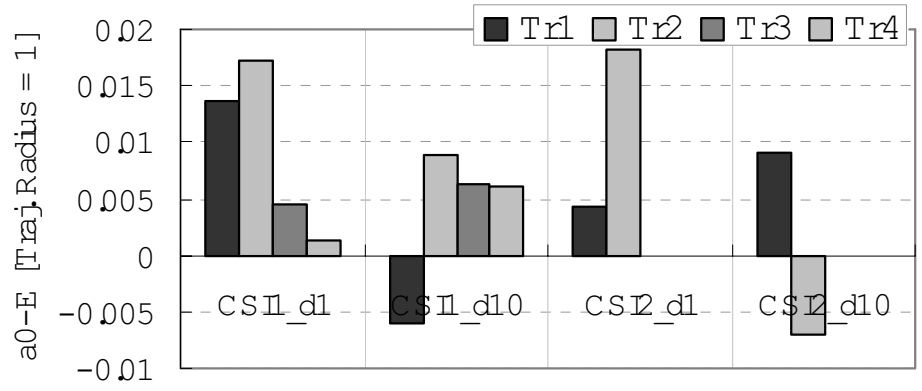

Fig. 8. The error of the norm circle's radius to the target trajectory's radius in each exercise session of the CSCI subjects. Left two are the first subject's and right two are the second subject's results. The $\mathrm{d} 1$ in the figure are for the 1st day's data and d10 are for the 10th days data. $\operatorname{Tr} 1$ through $\operatorname{Tr} 4$ are the order of the exercise session in each day.

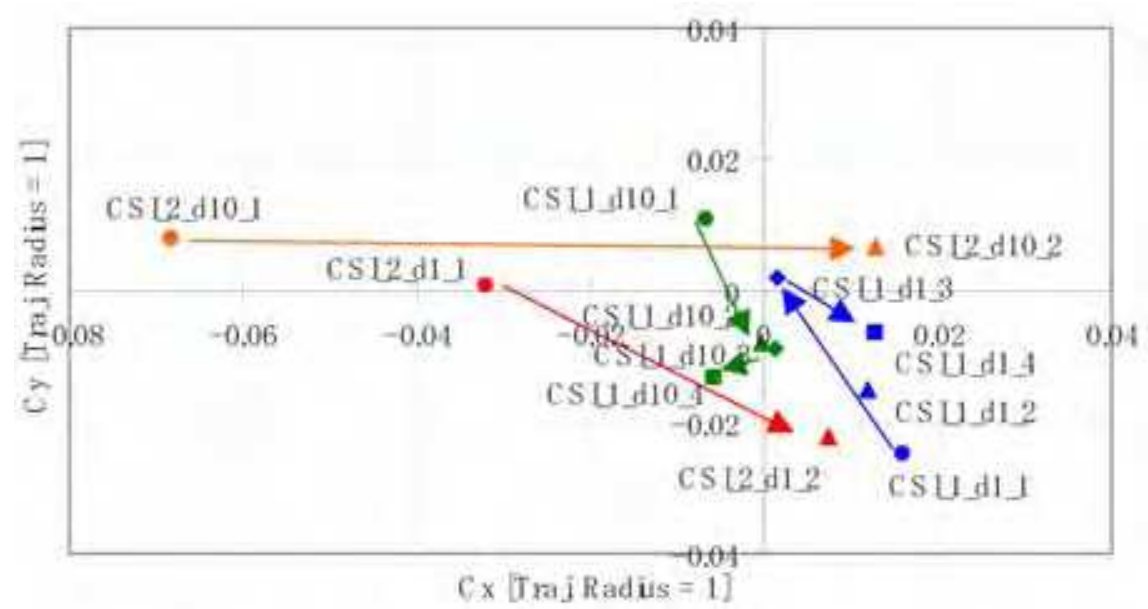

Fig. 9. The eccentric relation of the norm circle in each exercise session. The arrows describe the time variance of the norm circle center within the session. The first and second CSCI subject's data are presented as CSI1 and CSI2, accordingly. The 1st day's data is shown as d1 and 10th day's data as d10. The trials are described as _ 1 to _ 4 . 

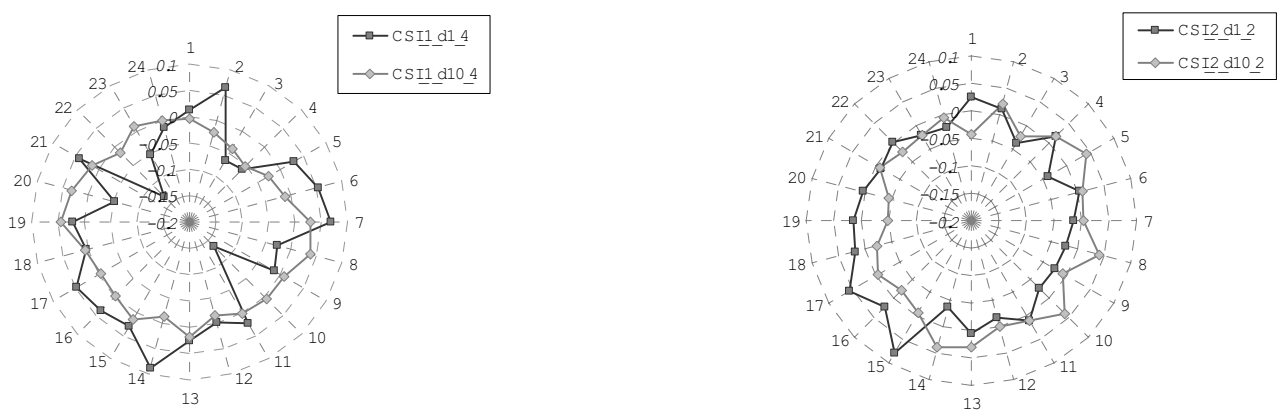

Fig. 10. The distortion of the marker trajectory to the norm circle. Left is the result of the first CSCI subject and the right is the result of the second CSCI subject. The numbers 1 to 24 describes the points in 15- degrees increment from the starting position around the target trajectory.

\section{Future Research}

Based on our experience with our subjects the scores presented after each unit session strongly affect the subject's motivation. The subjects were first very pleased with the scores being higher then they expected. However, our simple scoring algorithm based on the error distance rating was not sensitive to the gradual performance changes they were making. The mismatch of the score and what they sensing on their performance improvement has caused less interest of continuing the exercise. The computational results show that indices of harmonic analysis can extract feature of the subject's performance quantitatively. Therefore, these indices may help to set an adequate goal for the subjects to attempt. As an example, matching the norm circle radius to the target trajectory can be a short time goal to begin with, and then the eccentric relation. If we gain further understanding of the exercise effect on the neuromuscular function, the goals can be established based on previous successful rehabilitation cases or target tasks that the subject aims of achieving. As an example, a participant who joined the test earlier commented that the training helped him to regain stability of driving with a steering wheel. An exercise scenario can be created to reinforce such task accomplishment.

To enhance further understanding of the recovery of the upper limb function in spinal cord and peripheral injuries, utilizing electromyogram (EMG) recordings in parallel with the joystick device will be useful. Calacie's group showed that EMG recording was more accurate for predicting contractile force within multiple muscle groups at both acute and chronic stages following spinal cord injury. And with the EMG recording system, they had found that recovery of voluntary contraction in motor-incomplete injury tends to occur after 5 week post-injury, which are post hospital discharge (Calacie et al., 2004). The disadvantage of the EMG method is that the system is expensive and requires time consuming setup. With these conditions, it is unlikely to be used at home or local clinics, therefore easier method such as our system to confirm the timing of recovery will be beneficial. Further study to correlate the EMG activities with force-based positioning will be needed.

Further research is needed for developing an automatic adjustment function in the exercise scenanrio. Target speed and marker's response to the applied force should be automatically 
tweaked based on feeding back performance score. In our testing setups, the marker's response to the force, i.e. marker gain, was adjusted to the individual with trial and error. By applying Jacobs' result in (Jacobs et al., 2004), where the peak and mean power loading differed from the level of injury and the subject's body mass, it would be superior to create a database to automate or ease the selection in the initial adjustment. Method based on evidence should be more reasonable for the medical staffs and would be a better interface than numerically or graphically setting the parameters. Furthermore, it would assist the evaluation with proper bias on comparing the data with different level of functional disability.

Further study on advanced method for robust multidimensional data analysis is needed for these devices to be more useful in the clinical condition where the subjects have wide variety of disability and neuromuscular function is unstable. A methodology with higher accuracy and sensitivity without adding additional cost for the diagnosis will be an indispensable requisites to develop such device at a suitable scale. We believe MahalanobisTaguchi System will be one of the key tools to implement (Kanbayashi et al., 2006). This evaluation method creates an unit distance scale from the similarity among the ideal or healthy data constellation. Mahalanobis distance is computed as an level of anomalousness or the degree of severity. When the result is larger than a single unit on the scale, the distance from the center is presented as the tested data's abnormality. Therefore, the prominent the abnormal condition is the greater the computed distance will become. To implement this method, data from an able-bodied subjects are collected to create the scale. Then the different level of CSCI and peripheral nerve disorder subject's should be evaluated to correlate the scale with the functional limitation. By discussing the distribution of the subjects' performance score of the clinically distinctive level and equivocal level, the accuracy and sensitivity can be verified.

\section{Conclusion}

The research backgrounds and the design concepts of a simple take-home system for upper limb rehabilitation of the cervical spinal cord and peripheral nerve injured subjects are presented. A force-based target tracking task is selected for application. A method for evaluating the performance on a circular locus is presented and the result of implementing the algorithm is described. Using the harmonic analysis method quantifies the geometric character of the locus and adds sights to understand subject's performance in a quantitative manner. Our result are limited due to small number of subjects and further experiments are needed. Further work is needed to create a exercising program scenario based on quantitative and subjective indices and clinical testing.

\section{References}

Amirabdollahian, F.; Loureiro, R.; Driessen, B. \& Harwin, W. (2001). Error correction movement for machine assisted stroke rehabilitation, In: Integration of Assistive Technology in the Information Age, Mokhtari, M. (Ed.), 45-59, IOS Press, ISBN1-58603171-6, Amsterdam

Calancie, B.; Molano, M.R. \& Broton, J.G. (2004). EMG for assessing the recovery of voluntary movement after acute spinal cord injury in man, Clinical Neurophysiology, 115, 1748-1759, ISSN 1388-2457 
Chelette, T.L.; Repperger, D.W. \& Phillips C.A. (1995). Enhanced metrics for identification of forearm rehabilitation, IEEE Transactions on Rehabilitation Engineering, Vol. 3, No. 1, 122-131, ISSN1063-6528

Coote, S.; Stokes, E.; Murphy, B. \& Harwin, W. (2003). The effect of GENTLE/s robotmediated therapy on upper extremity dysfunction post stroke, Proceedings of the 8 th International Conference on Rehabilitation Robotics, 59-61, ISBN89-88366-09-3, Daejeon, Korea, Apr., 2003, HWRS-ERC, Daejeon

Furusho, J.; Koyanagi, K.; Ryu, U.; Inoue, A. \& Oda, K. (2003). Development of rehabilitation robot system with functional fluid devices for upper limbs, Proceedings of the 8 th International Conference on Rehabilitation Robotics, 31-34, ISBN89-88366-09-3, Daejeon, Korea, Apr., 2003, HWRS-ERC, Daejeon

Goto, E.; Ohnishi, K.; Miyagawa, H. \& Saito Y. (2005). Field test of a force control rehabilitation system for quantitative evaluation of the disorder in the upper extremities, Proceedings of the 2005 IEEE 9th International Conference on Rehabilitation Robotics, 82-85, ISBN0-7803-9004-0, Chicago, IL, USA, June, 2005, Omnipress

Haugland, M.; Lickel, A.; Haase, J. \& Sinkjær, T. (1999) Control of FES thumb force using slip information obtained from the cutaneous electroneurogram in quadriplegic man, IEEE Transactions on Rehabilitation Engineering, Vol. 7, No. 2, (June) 215-226, ISSN1063-6528

Hogan, N; Krebs, H.I.; Rohrer, B.; Palazzolo, J.J.; Dipietro, L.; Fasoli, S.E.; Stein, J; Hughes, R; Frontera, W.R.; Lynch, D. \& Volpe, B.T. (2006). Motions or muscles? Some behavioural factors underlying robotic assistance for motor recovery, Journal of Rehabilitation Research \& Development, Vol. 43, No. 5, (Aug./Sept.) 605-618, ISSN 0748-7711

Ide, M.; Fujiie, K; Mitarai, K. \& Kurosu, K. (1995). Operational characteristics of joystick with single-speed floating action on cervical injuries, The Japanese Journal of Ergonomics, Vol. 31, No. 2, 141-149

Jacobs, P.L.; Johnson, B.M.; Mahoney, E.T.; Carter, A.B. \& Somarroba, G.A. (2004). Effect of variable loading in the determination of upper-limb anaerobic power in persons with tetraplegia, Journal of Rehabilitation Research \& Development, Vol. 41, No. 1, (Jan./Feb.) 9-14, ISSN 0748-7711

Kahn, L.E.; Averbuch, M.; Rymer, W.Z. \& Reinkensmeyer, D.J. (2001). Comparison of robotassisted reaching to free reaching in promoting recovery from chronic stroke, In: Integration of Assistive Technology in the Information Age, Mokhtari, M. (Ed.), 39-44, IOS Press, ISBN1-58603-171-6, Amsterdam

Kanbayashi, Y.; Saito, Y.; Yano, H. \& Ohnishi, K. (2006). Evaluation study of an arm training system for patients with cervical spinal cord injuries, Quality Engineering, Vol. 14, No. 3, (June 2006) 69-74 (in Japanese)

Krebs, H.I.; Hogan, N.; Aisen, M.L. \& Volpe, B.T. (1998). Robot-aided neurorehabilitation, IEEE Transactions on Rehabilitation Engineering, Vol. 6, No. 1, 7587, ISSN1063-6528

Krebs, H.I.; Volpe, B.T.; Palazzolo, J.; Rohrer, B.; Ferraro, M.; Fasoli, S.; Edelstein, L. \& Hogan, N. (2001). Robot-aided neuro-rehabilitation in stroke: interim results on the follow-up of 76 patients and on movement performance indices, In: Integration of Assistive Technology in the Information Age, Mokhtari, M. (Ed.), 45-59, IOS Press, ISBN1-58603-171-6, Amsterdam 
Latimer, A.E.; Martin Ginis, K.A.; Hicks, A.L. \& McCartney N. (2004). An examination of the mechanisms of exercise-induced change in psychology well-being among people with spinal cord injury, Journal of Rehabilitation Research \& Development, Vol. 41, No. 5, (Sept./Oct.) 643-652, ISSN 0748-7711

Lenze, E.J.; Munin, M.C.; Quear, T.; Dew, M.A.; Rogers J.C.; Begley, A.E. \& Reynolds III, C.F. (2004). Significance of poor patient participation in physical and occupational therapy for functional outcome and length of stay, Archives of Physical Medicine and Rehabilitation, Vol. 85, (Oct) 1599-1601, ISSN 0003-9993

Murayama, N. (2002). Technical approach for rehabilitation medicine: development of a quantitative analysis system for voluntary movement functions in the upper limb, Japanese Journal of Rehabilitation Medicine, Vol. 39, No. 11, 735-743 (in Japanese)

Nakata, T. (1972). Engineering Analysis, Ohm, Tokyo (in Japanese)

Ohnishi, K. ; Goto, E.; Ikeuchi, H.; Imado, K.; Miyagawa, H. \& Saito, Y. (2005). Development testing of a force control rehabilitation system for disorder in the upper extremities, Proceedings of the First International Conference On Complex Medical Engineering, 504509, ISBN4-9902522-0-9, Takamatsu, Japan, May, 2005

Ohnishi, K.; Goto, E.; Sugiki, F.; Imado, K.; Ikeuchi, H.; Kito, N.; Miyagawa, H. \& Saito, Y. (2004). Home-use upper limb rehabilitation device for cervical spinal cord injured patients, In: Computers helping people with special needs, Miesenberger, K.; Klaus, J.; Zagler, W. \& Burger, D. (Eds.), 880- 888, Springer-Verlag, ISBN3-540-22334-7, Berlin Heidelberg.

Ohnishi, K.; Miyagawa, H.; Negoto, H. \& Saito, Y. (2003). Rehabilitation system for arm-eye coordination of the palsy arm, Proceedings of 6th Japan-France Congress on Mechatronics and 4th Asia-Europe Congress on Mechatronics, 117-122, Hatoyama, Saitama, Japan, Sept, 2003

Popovic, M.R.; Popovic, D.B. \& Keller, T. (2002) Neuroprostheses for grasping, Neurological Research, Vol. 24, (July) 443-452, ISSN0161-6412

Prange, G.B.; Jannink, M.J.A.; Groothuis-Oudshoorn, C.G.M.; Hermens, H.J. \& IJzerman M.J. (2006). Systematic review of the effect of robot-aided therapy on recovery of the hemiparetic arm after stroke, Journal of Rehabilitation Research \& Development, Vol. 43, No. 2, (Mar./ Apr.) 171-184, ISSN 0748-7711

Reinkensmeyer, D.J. (2003). Chapter 35 Rehabilitators, In: Standard handbook of biomedical engineering $\mathcal{E}$ design, Kutz, M. (Ed.), 35.1-35.17, McGraw-Hill, ISBN0-07-135637-1, New York

Reinkensmeyer, D.J.; Hogan, N; Krebs, H.I.; Lehman, S.L. \& Lum, P.S. (2000). 38 Rehabilitators, robots, and guides: new tools for neurological rehabilitation, In: Biomechanics and neural control of posture and movement, Winters, J.M. \& Crago, P.E. (Eds.), 516-533, Springer-Verlag, ISBN0-387-94974-7, New York

Shingu, H.; Ohama, M.; Ikata, T.; Katoh, S. \& Akatsu, T. (1995). A nationwide epidemiological survey of spinal cord injuries in Japan from January 1990 to December 1992, Paraplegia, Vol. 33-4, 183-188, ISSN 0031-1758

Shor, P.C.; Lum, P.S.; Burgar, C.G.; Van der Loos, H.F.M.; Majmundar, M. \& Yap. R. (2001) The effect of robotic-aided therapy on upper extremity joint passive range of motion and pain. In: Integration of Assistive Technology in the Information Age, Mokhtari, M. (Ed.), 79-83, IOS Press, ISBN1-58603-171-6, Amsterdam

Sumida, M.; Tokuhiro, A.; Magara, A.; Toyonaga, T. \& Uchida, R. (Eds.), (2001). Clinical outcome of spinal cord injury, Ishiyaku Publishers, ISBN4-263-21125-1, Tokyo (In Japanese) 
Takahashi, Y.; Terada, T.; Inoue, K.; Ito, Y.; Lee, H. \& Komeda, T. (2003). Upper-limb rehabilitation exercises using haptic device system, International Journal of Humanfriendly Welfare Robotic Systems, Vol.4, No.2, 18-22

Takaoka, T.; Wakabayashi, H. \& Ito, T. (1999). Rehabilitation for home-bound elderly disabled with spinal cord injury, Journal of Clinical Rehabilitation, Vol. 8, No. 10, 943950 (In Japanese)

Wyndaele, M. \& Wyndaele, J-J. (2006) Incidence, prevalence and epidemiology of spinal cord injury: what learns a worldwide literature survey? Spinal Cord. Vol. 44-9, 523508, ISSN 1362-4393 


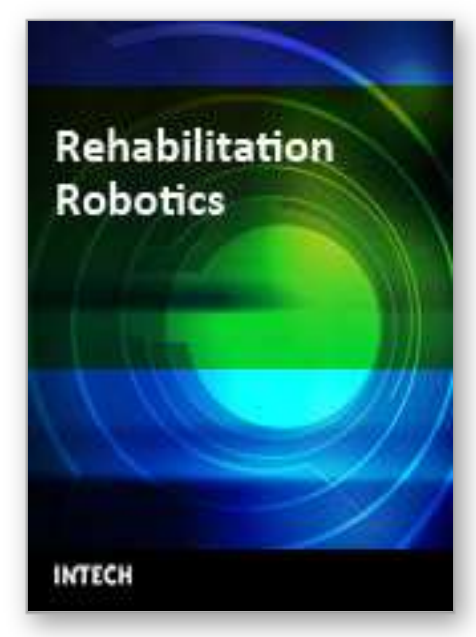

\author{
Rehabilitation Robotics \\ Edited by Sashi S Kommu
}

ISBN 978-3-902613-04-2

Hard cover, 648 pages

Publisher I-Tech Education and Publishing

Published online 01, August, 2007

Published in print edition August, 2007

The coupling of several areas of the medical field with recent advances in robotic systems has seen a paradigm shift in our approach to selected sectors of medical care, especially over the last decade.

Rehabilitation medicine is one such area. The development of advanced robotic systems has ushered with it an exponential number of trials and experiments aimed at optimising restoration of quality of life to those who are physically debilitated. Despite these developments, there remains a paucity in the presentation of these advances in the form of a comprehensive tool. This book was written to present the most recent advances in rehabilitation robotics known to date from the perspective of some of the leading experts in the field and presents an interesting array of developments put into 33 comprehensive chapters. The chapters are presented in a way that the reader will get a seamless impression of the current concepts of optimal modes of both experimental and ap- plicable roles of robotic devices.

\title{
How to reference
}

In order to correctly reference this scholarly work, feel free to copy and paste the following:

Kengo Ohnishi, Keiji Imado, Yukio Saito and Hiroomi Miyagawa (2007). Upper Limb Rehabilitation System for Self-Supervised Therapy: Computer-Aided Daily Performance Evaluation for the Trauma and Disorder in the Spinal Cord and Peripheral Nerves, Rehabilitation Robotics, Sashi S Kommu (Ed.), ISBN: 978-3-902613-04-2, InTech, Available from:

http://www.intechopen.com/books/rehabilitation_robotics/upper_limb_rehabilitation_system_for_selfsupervised_therapy_computer-aided_daily_performance_evalu

\section{INTECH}

open science | open minds

\author{
InTech Europe \\ University Campus STeP Ri \\ Slavka Krautzeka 83/A \\ 51000 Rijeka, Croatia \\ Phone: +385 (51) 770447 \\ Fax: +385 (51) 686166 \\ www.intechopen.com
}

\author{
InTech China \\ Unit 405, Office Block, Hotel Equatorial Shanghai \\ No.65, Yan An Road (West), Shanghai, 200040, China \\ 中国上海市延安西路65号上海国际贵都大饭店办公楼 405 单元 \\ Phone: +86-21-62489820 \\ Fax: +86-21-62489821
}


(C) 2007 The Author(s). Licensee IntechOpen. This chapter is distributed under the terms of the Creative Commons Attribution-NonCommercial-ShareAlike-3.0 License, which permits use, distribution and reproduction for non-commercial purposes, provided the original is properly cited and derivative works building on this content are distributed under the same license. 